\title{
Le Forum Datenaustausch adopte une solution consensuelle
}

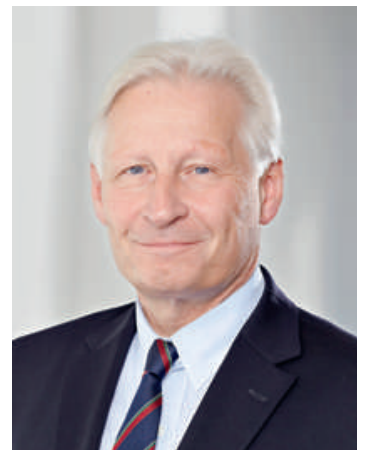

Fin 2011, le Parlement a décidé que les hôpitaux devaient faire figurer sur les factures établies dans le cadre des DRG «les diagnostics et les procédures sous forme codée, conformément aux classifications contenues dans l'édition suisse correspondante publiée par le département compétent». Depuis lors se pose la question de la mise en ouvre de cette mesure. Le Forum Datenaustausch apporte dans un premier temps une réponse consensuelle en adoptant le standard XML4.4.

\section{Le moment d'un premier bilan critique est venu}

En juillet 2012, suite à de multiples interventions de la FMH, le Conseil fédéral a demandé aux assureurs de créer des services de réception des données certifiés, qui n'auront le droit de transmettre que les données de factures suspectes aux divisions de prestations des caisses. Pour la FMH comme pour les

\section{Une solution conjointe et consensuelle a pu être trouvée par le Forum Daten- austausch.}

organisations de patients, cela a marqué une étape importante dans la bonne direction. Par la suite, la FMH s'est investie à plusieurs niveaux pour que ne soient transmises que les données réellement nécessaires à l'affectation à un groupe de cas DRG ou à la vérification d'une facture. Après d'âpres négociations et des discussions approfondies, les instances impliquées, notamment le Forum Datenaustausch, ont trouvé un consensus pour la plupart des points en suspens. En revanche, la question du lieu de séjour avant et après une hospitalisation reste controversée, car ces informations ne sont pas nécessaires à la vérification d'une facture; elles permettent cependant de créer des enchaînements et des regroupements de cas. Sur ce point, c'est l'ordonnance du département de décembre 2012 qui a tranché; celle-ci diffère en effet des dispositions de l'OAMal et précise la livraison de données supplémentaires. Finalement, une solution conjointe et consensuelle a pu être trouvée en février 2013 par le Forum Datenaustausch.

\section{Pourquoi la FMH s'est-elle ici autant engagée en faveur du secret médical?}

S'engager en faveur de la protection des données, et en particulier en faveur du secret médical dans l'intérêt des patientes et des patients, quel que soit le lieu du traitement, demeure une tâche fondamentale de la FMH. Seules les données réellement nécessaires doivent circuler. Parmi les 50 diagnostics

\section{La FMH est attachée à ce que des solu- tions pragmatiques et consensuelles soient élaborées en matière de trans- missions des données.}

susceptibles d'être communiqués, beaucoup ne sont pas décisifs pour l'affectation à un DRG et donc aussi pour la vérification de la facturation. Dans ce contexte, la transmission des données signifierait non seulement une atteinte massive à la sphère privée des patientes et des patients mais elle pourrait aussi remettre en cause la relation de confiance entre le patient et son médecin traitant, également indispensable à la réussite d'un traitement. Ceci vaut aussi à l'hôpital où tant les médecins que l'administration sont responsables du maintien du secret médical. Là aussi, la FMH s'engage avec énergie pour défendre les intérêts des médecins hospitaliers et les soutenir. C'est d'ailleurs un engagement extrêmement important et nécessaire comme le confirment, dans le cas de la facturation, différents médecins hospitaliers ainsi que des représentants de patientes et de patients et même des collaborateurs hospitaliers chargés du codage.

\section{De «service de réception" à «service de vérification»} Ces réactions nous incitent à poursuivre nos efforts. La FMH sera en particulier très attentive à la manière dont les services de réception des données seront conçus; en effet, seuls les diagnostics et les procédures décisifs pour le groupage devront être transmis. Par ailleurs, la FMH est attachée à ce que des solutions pragmatiques et appropriées soient élaborées en concertation avec les partenaires du domaine de la santé. Son objectif principal consiste à ce que les «services de réception» des données deviennent des «services de vérification» neutres.

Dr Urs Stoffel, membre du Comité central de la FMH, responsable du domaine eHealth - infrastructure de sécurité 